\title{
Risk factors for low cardiac output syndrome in children with congenital heart disease undergoing cardiac surgery: a retrospective cohort study
}

Xinwei Du', Hao Chen ${ }^{1}$, Xiaoqi Song ${ }^{1}$, Shunmin Wang ${ }^{1}$, Zedong Hao ${ }^{2}$, Lifeng Yin ${ }^{2}$ and Zhaohui Lu*

\begin{abstract}
Background: Low cardiac output syndrome (LCOS) is an important complication of cardiac surgery. It is associated with increased morbidity and mortality. The incidence of LCOS after surgery is high in patients with congenital heart disease (CHD). Therefore, determining the risk factors of LCOS has clinical significance for the management of CHD. This study aimed to analyze the risk factors of LCOS.

Methods: We conducted a retrospective analysis of children with CHD who underwent cardiac surgery at Shanghai Children's Medical Center between January 1, 2014, and December 31, 2017. Demographic characteristics and baseline data were extracted from the health data resource center of the hospital, which integrates clinical routine data including medical records, diagnoses, orders, surgeries, laboratory tests, imaging, nursing, and other subsystems. Logistic regressions were performed to analyze the risk factors of LCOS.

Results: Overall, 8660 infants with CHD were included, and 864 (9.98\%) had LCOS after surgery. The multivariate regression analysis identified that age (OR $0.992,95 \% \mathrm{Cl}: 0.988-0.997, p=0.001)$, tricuspid regurgitation (1.192, $1.072-1.326, p=0.001)$, Risk Adjustment in Congenital Heart Surgery-1 risk grade (1.166, 1.011-1.345, $p=0.035)$, aortic shunt (left-to-right: 1.37, 1.005-1.867, $p=0.046$; bi-directional: 1.716, 1.138-2.587, $p=0.01$ ), atrial shunt (left-toright: 1.407, 1.097-1.805, $p=0.007$; right-to-left: 3.168, 1.944-5.163, $p<0.001$; bi-directional: 1.87, 1.389-2.519, $p<$ 0.001), ventricular level shunt (left-to-right: $0.676,0.486-0.94, p=0.02$; bi-directional: $2.09,1.611-2.712, p<0.001$ ), residual shunt $(3.489,1.502-8.105, p=0.004)$, left ventricular outflow tract obstruction $(3.934,1.673-9.254, p=0.002)$, right ventricular outflow tract obstruction $(3.638,1.225-10.798, p=0.02)$, circulating temperature (mild hypothermia: 1.526, 95\% Cl: 1.205-1.934, $p<0.001$; middle and low temperature: 1.738, 1.236-2.443, $p=0.001$ ), duration of cardiopulmonary bypass $(1.009,1.006-1.012, p<0.001)$, myocardial preservation using histidine-tryptophanketoglutarate $(1.677,1.298-2.167, p<0.001)$, and mitral insufficiency $(1.714,1.239-2.37, p<0.001)$ were independent risk predictors of LCOS.
\end{abstract}

Conclusions: The incidence of postoperative LCOS in CHD children remains high. Circulation temperature, myocardial preservation using histidine-tryptophan-ketoglutarate, and usage of residual shunt after surgery were independent risk predictors for LCOS.

Keywords: Low cardiac output syndrome, Congenital heart disease, Risk factor, Cardiac surgery, Left ventricular ejection fraction, Cardiopulmonary bypass

\footnotetext{
* Correspondence: luzhaohui@scmc.com.cn

'Department of Cardiothoracic Surgery, Shanghai Children's Medical Center,

School of Medicine, Shanghai Jiao Tong University, 1678 Dongfang Road,

Pudong district, Shanghai, China

Full list of author information is available at the end of the article
}

(c) The Author(s). 2020 Open Access This article is distributed under the terms of the Creative Commons Attribution 4.0 International License (http://creativecommons.org/licenses/by/4.0/), which permits unrestricted use, distribution, and reproduction in any medium, provided you give appropriate credit to the original author(s) and the source, provide a link to the Creative Commons license, and indicate if changes were made. The Creative Commons Public Domain Dedication waiver (http://creativecommons.org/publicdomain/zero/1.0/) applies to the data made available in this article, unless otherwise stated. 


\section{Background}

Low cardiac output syndrome (LCOS) is the most common complication following cardiac surgeries. The incidence of LCOS in the surgical treatment of congenital heart disease (CHD) has been reported to be $25-60 \%$ [1], associated with a significant risk of mortality among patients [2,3]. LCOS is associated with a decreased ejection fraction and decreased oxygen supply, which may cause hypoxia. The patients are often at a high risk of mortality and require more intensive care such as extended stay in the intensive care unit (ICU) and ventilatory support.

The etiology of postoperative LCOS is multifactorial [4]. Pathologically, endothelial dysfunction and myocardial stunning, acute changes in the loading conditions of the myocardium, use of cardioplegia, activation of the inflammatory and complement cascade caused by cardiopulmonary bypass $(\mathrm{CPB})$, and the residual hemodynamic burden of uncorrected defects [5] have been suggested to be the causes of LCOS. The most commonly reported clinical predictors of LCOS include left ventricular ejection fraction $<20 \%$, surgical history, female gender, and increasing age $[2,6,7]$. Prediction models based on clinical data, such as EuroSCORE, have been proposed [8]. However, the risk factors of LCOS are varied in the literature according to the population and surgery categories included [2, 9-11].

To date, only a few studies presented the detailed analysis of the risk factors for postoperative LCOS and the further complications in children with CHD. This study aimed to analyze the risk factors for LCOS and to determine their association with the incidence of postoperative death, extended ICU stay, and the duration of mechanical ventilator support in Chinese children with and without LCOS.

\section{Methods}

\section{Study design and population}

In this retrospective study, patients with $\mathrm{CHD}$ who underwent cardiac surgery at Shanghai Children's Medical Center from January 1, 2014, to December 31, 2017, were included. Patients aged $\geq 18$ years, on $\mathrm{CPB}$, who had undergone general thoracic surgery not involving cardiotomy, or with uncertain in-hospital survival records were excluded from the study. The study was approved by the Ethical Committee of Shanghai Children's Medical Center. Demographic characteristics and baseline data were extracted from the health data resource center of the hospital, which integrated clinical routine data including medical records, diagnoses, orders, operations, laboratory tests, imaging, nursing, and other subsystems.

Patients were classified into two groups, based on whether they developed LCOS into the LCOS and non-LCOS groups. The baseline characteristics and intraoperative features of the two groups were compared. Associations of these characteristics with LCOS were analyzed using regression analysis. In addition, the mortality, extended ICU stay, and duration of mechanical ventilator support were compared between the two groups.

\section{Outcomes}

LCOS is characterized by clinical signs or symptoms including elevated blood lactate or rapid increase in blood lactate, decreased central venous oxygen saturation, increased arterial to central venous oxygen saturation difference, decreased urine output, increased peripheral skin temperature to core body temperature difference, and low echocardiographic Doppler-derived cardiac index, high inotrope requirement [12]. In-hospital mortality was defined as death during the same hospitalization regardless of cause. Prolonged ICU stay was defined as $>3$ days of ICU stay, and extended duration of mechanical ventilation support was defined as $>48 \mathrm{~h}$ ' ventilatory support [13].

\section{Risk factors}

The risk factors of LCOS analyzed in this study include baseline and demographic data, pre-operative Doppler echocardiography characteristics, characteristics of surgery and $\mathrm{CPB}$, as well as postoperative measures of $\mathrm{CHD}$. The body mass index (BMI), as categorical data percentiles varied according to age groups, which were age-adjusted according to the pediatric BMI reference data for China [14]. Moreover, the Risk Adjustment in Congenital Heart Surgery-1 (RACHS-1) score was defined as ordinal data; an increase in the RACHS-1 score can indicate the risk of LCOS [15]. Nutrition status has been recognized as an important risk factor of postoperative complications in CHD surgery. In this study, the incidences of LCOS in CHD children with different nutrition status were analyzed separately.

\section{Statistical analysis}

Continuous variables are expressed as mean \pm standard deviation (SD) (normally distributed) or median (IQR) (non-normally distributed). Categorical variables are presented as frequency (\%). Depending on the distributions, the t-test or Mann-Whitney U test was used to compare the continuous variables between the groups. In addition, a chi-squared test or Fisher's exact test was used for the comparison of categorical variables.

Univariate logistic regressions with LCOS as an outcome were analyzed first. Then, the Variance Inflation Factor was calculated to explore the independence of the selected variables. The results are listed in Supplemental Table 1, and there is no evidence to show dependence among the selected factors. Therefore, the significant variables were entered into multiple logistic regressions without an interaction term, and the stepwise variable 
selection method was used to identify the potential risk factors of LCOS. All tests were two-sided, and $P<0.05$ was considered as statistically significant. All analyses were performed using SAS software, version 9.4 (SAS Institute INC).

\section{Results}

\section{Demographic characteristics of CHD children}

Overall, 8660 children were included in the study, with 864 (9.98\%) LCOS cases after surgery. The demographic characteristics at baseline including age $(p<0.001)$ and body mass index $(\mathrm{BMI})<5$ th percentile $(p=0.016)$ were significantly different between the CHD children with LCOS and those without LCOS (Table 1).

\section{Preoperative Doppler echocardiography characteristics of CHD children}

When Doppler echocardiography characteristics were compared between the CHD children in the two groups, it was found that aortic shunt $(p<0.001)$, atrial shunt $(p<0.001)$, ventricular level shunt $(p<0.001)$, aortic insufficiency $(p=0.035)$, pulmonary insufficiency $(p=0.007)$, tricuspid regurgitation (TR) $(p=0.001)$, and mitral insufficiency $(p=0.044)$ of LCOS patients were significantly different from those of non-LCOS patients (Table 1).

\section{Characteristics of surgery and cardiopulmonary bypass of CHD children}

In LCOS patients, operation characteristics $(p<0.001)$, procedure complexity (represented by RACHS-1 risk grade) $(p<0.001)$, CPB duration $(p<0.001)$, aortic clamping time $(<0.001)$, history of heart surgery $(p<0.001)$, circulating temperature $(p<0.001)$, circulation method $(<0.001)$, and myocardial preservation $(p<0.001)$ were significantly different from those without LCOS (Table 1).

\section{Postoperative measures of CHD children}

There were significant differences in postoperative residual shunt $(p<0.001)$, left ventricular outflow tract obstruction (LVOTO, $p<0.001$ ), right ventricular outflow tract obstruction (RVOTO, $p<0.001$ ), and postoperative rhythm of the heart $(p<0.001)$ between LCOS and nonLCOS patients (Table 1).

\section{Logistic regression analysis}

Univariate analysis identified that age, aortic insufficiency, pulmonary insufficiency, TR, RACHS-1 risk grade, aortic shunt, atrial shunt, ventricular level shunt, emergency extracorporeal surgery, history of heart surgery, residual shunt, LVOTO, RVOTO, abnormal circulation temperature, circulation method (except for parallel $\mathrm{CPB}$ and cardiocerebral perfusion), duration of $\mathrm{CPB}$, aortic clamping time, postoperative rhythm of the heart (III $\left.\mathrm{AVB}, \mathrm{II}^{\circ} \mathrm{AVB}\right)$, difficult to wean from $\mathrm{CPB}$, myocardial preservation using histidine-tryptophan-ketoglutarate (HTK), BMI $<5$ th percentile, and mitral insufficiency were significantly associated with LCOS in CHD children (Table 2).

Multivariate analysis of these variables found age (OR 0.992, 95\% CI: 0.988-0.997, $p=0.001$ ), TR (OR 1.192, 95\% CI 1.072-1.326, $p=0.001$ ), RACHS-1 risk grade (OR 1.166, 95\% CI 1.011-1.345, $p=0.035$ ), aortic shunt (left-to-right: OR 1.37, 95\% CI 1.005-1.867, $p=0.046$; bi-directional: OR 1.716, 95\% CI 1.138-2.587, $p=0.01$ ), atrial shunt (left-to-right: OR 1.407, 95\% CI 1.097-1.805, $p=0.007$; right-to-left: OR $3.168,95 \%$ CI 1.944-5.163, $p<$ 0.001; bi-directional: OR 1.87, 95\% CI 1.389-2.519, $p<$ 0.001 ), ventricular level shunt (left-to-right: OR 0.676, $95 \%$ CI $0.486-0.94, p=0.02$; bi-directional: OR $2.09,95 \% \mathrm{CI}$ $1.611-2.712, p<0.001$ ), residual shunt (OR $3.489,95 \% \mathrm{CI}$ $1.502-8.105, p=0.004$ ), LVOTO (OR 3.934, 95\% CI $1.673-9.254, p=0.002$ ), RVOTO (OR 3.638, 95\% CI 1.225-10.798, $p=0.02$ ), circulating temperature (mild hypothermia: OR 1.526, 95\% CI 1.205-1.934, $p<0.001$; middle and low temperature: OR 1. 738, 95\% CI 1.2362.443, $p=0.001$ ), duration of CPB (OR 1.009, 95\% CI 1.006-1.012, $p<0.001)$, myocardial preservation using HTK (OR 1.677, 95\% CI 1.298-2.167, $p<0.001$ ), and mitral insufficiency (OR 1.714, 95\% CI 1.239-2.37, $p<0.001$ ) were independent risk predictors of LCOS (Table 2).

\section{Postoperative mortality, prolonged ICU stay, and extended medical ventilator support in LCOS and non- LCOS CHD children}

The mortality rate $(7.18 \%$ vs. $1.08 \%, p<0.001)$, ICU stay $>3$ days $(89.24 \%$ vs. $33.35 \%, p<0.001)$, and duration of mechanical ventilator support $>48 \mathrm{~h}(93.21 \%$ vs. $44.5 \%$, $p<0.001$ ) of LCOS children were significantly higher than those of non-LCOS children (Table 3).

Incidence of postoperative LCOS in children with CHD of different nutritional status

In CHD children with $\mathrm{BMI}<5$ th percentile, the incidences of LCOS showed a gradually decreasing trend in neonates aged $>3$ months. In neonates aged 3-6 months, the incidence increased, declined in children aged 6 months to $>3$ years old. In patients with BMI 5th 95th percentile and $>95$ th percentile, the incidences of LCOS showed a gradually decreasing trend. (Table 4 ).

\section{Discussion}

In our study, the incidence of LCOS was 9.98\%, which is higher than that in adult patients postoperatively (2.4$9.1 \%)[2,3,7]$, but lower than that of another study on newborns, in which the LCOS incidence at $36 \mathrm{~h}$ after surgery was $25.9,17.5$, and $11.7 \%$ of infants (median age, 3 months) on placebo, low-dose milrinone, and highdose milrinone, respectively [16]. In a secondary retrospective analysis of a prospective randomized trial, 
Table 1 The clinical characteristics of CHD children (including demographic characteristics, pre-operative color doppler echocardiography characteristics, the characteristic of surgery and cardiopulmonary bypass, and post-operative characteristics)

\begin{tabular}{|c|c|c|c|c|c|}
\hline \multirow{2}{*}{\multicolumn{2}{|c|}{$\frac{\text { Parameter }}{\text { Demographic characteristics }}$}} & \multirow[t]{2}{*}{$\operatorname{LCOS}(n=864)$} & \multirow[t]{2}{*}{ non-LCOS $(n=7796)$} & \multirow[t]{2}{*}{ Total $(n=8660)$} & \multirow[t]{2}{*}{$P$ value } \\
\hline & & & & & \\
\hline Age (days) & Median (IQR) & $206.5(94,507.5)$ & $350(182,950.5)$ & $329(173,916)$ & $<0.001$ \\
\hline \multirow[t]{2}{*}{ Gender } & MALE & $56.4 \%$ & $55.0 \%$ & $55.1 \%$ & \multirow[t]{2}{*}{0.4} \\
\hline & FEMALE & $43.6 \%$ & $45.1 \%$ & $44.9 \%$ & \\
\hline \multirow[t]{3}{*}{ BMI } & $<5$ th percentile & $23.4 \%$ & $19.0 \%$ & $19.5 \%$ & \multirow[t]{3}{*}{0.016} \\
\hline & 5th 95th percentile & $62.8 \%$ & $66.7 \%$ & $66.3 \%$ & \\
\hline & $>95$ th percentile & $13.8 \%$ & $14.3 \%$ & $14.2 \%$ & \\
\hline \multicolumn{6}{|c|}{ Pre-operative color doppler echocardiography characteristics } \\
\hline \multirow[t]{4}{*}{ Aortic shunt } & None & $76.1 \%$ & $90.4 \%$ & $89.0 \%$ & \multirow[t]{4}{*}{$<0.001$} \\
\hline & Left-to-right & $15.3 \%$ & $7.7 \%$ & $8.4 \%$ & \\
\hline & Right-to-left & $0.7 \%$ & $0.03 \%$ & $0.1 \%$ & \\
\hline & Bi-directional & $8.0 \%$ & $1.9 \%$ & $2.5 \%$ & \\
\hline \multirow[t]{4}{*}{ Atrial shunt } & None & $21.7 \%$ & $36.5 \%$ & $35.1 \%$ & \multirow[t]{4}{*}{$<0.001$} \\
\hline & Left-to-right & $39.6 \%$ & $47.8 \%$ & $47.0 \%$ & \\
\hline & Right-to-left & $6.9 \%$ & $2.7 \%$ & $3.1 \%$ & \\
\hline & Bi-directional & $31.8 \%$ & $13.0 \%$ & $14.9 \%$ & \\
\hline \multirow[t]{4}{*}{ Ventricular level shunt } & None & $23.7 \%$ & $28.6 \%$ & $28.1 \%$ & \multirow[t]{4}{*}{$<0.001$} \\
\hline & Left-to-right & $12.3 \%$ & $40 \%$ & $37.3 \%$ & \\
\hline & Right-to-left & $0.3 \%$ & $0.1 \%$ & $0.1 \%$ & \\
\hline & Bi-directional & $63.8 \%$ & $31.3 \%$ & $34.5 \%$ & \\
\hline \multirow[t]{7}{*}{$\mathrm{Al}$} & Negative & $80.0 \%$ & $83.1 \%$ & $82.8 \%$ & \multirow[t]{7}{*}{0.035} \\
\hline & Minimal & $13.6 \%$ & $10.8 \%$ & $11.0 \%$ & \\
\hline & Mild & $5.2 \%$ & $5.2 \%$ & $5.2 \%$ & \\
\hline & Mild-to-moderate & $0.3 \%$ & $0.5 \%$ & $0.4 \%$ & \\
\hline & Moderate & $0.4 \%$ & $0.3 \%$ & $0.3 \%$ & \\
\hline & Moderate-to-severe & $0.1 \%$ & $0.1 \%$ & $0.1 \%$ & \\
\hline & Severe & $0.5 \%$ & $0.1 \%$ & $0.1 \%$ & \\
\hline \multirow[t]{7}{*}{ MR } & Negative & $26.6 \%$ & $22.6 \%$ & $23.0 \%$ & \multirow[t]{7}{*}{0.9} \\
\hline & Minimal & $43.0 \%$ & $49.0 \%$ & $48.4 \%$ & \\
\hline & Mild & $18.5 \%$ & $20.5 \%$ & $20.3 \%$ & \\
\hline & Mild-to-moderate & $6.1 \%$ & $4.5 \%$ & $4.6 \%$ & \\
\hline & Moderate & $3.3 \%$ & $2.3 \%$ & $2.4 \%$ & \\
\hline & Moderate-to-severe & $1.7 \%$ & $0.7 \%$ & $0.8 \%$ & \\
\hline & Severe & $0.8 \%$ & $0.4 \%$ & $0.4 \%$ & \\
\hline \multirow[t]{7}{*}{ PI } & Negative & $16.3 \%$ & $9.2 \%$ & $9.9 \%$ & \multirow[t]{7}{*}{0.007} \\
\hline & Minimal & $53.1 \%$ & $59.5 \%$ & $58.9 \%$ & \\
\hline & Mild & $28.8 \%$ & $29.6 \%$ & $29.6 \%$ & \\
\hline & Mild-to-moderate & $0.7 \%$ & $0.6 \%$ & $0.6 \%$ & \\
\hline & Moderate & $1.1 \%$ & $1.0 \%$ & $1.0 \%$ & \\
\hline & Moderate-to-severe & $0 \%$ & $0.03 \%$ & $0.03 \%$ & \\
\hline & Severe & $0 \%$ & $0.04 \%$ & $0.04 \%$ & \\
\hline \multirow[t]{2}{*}{ TR } & Negative & $1.2 \%$ & $0.4 \%$ & $0.5 \%$ & \multirow[t]{2}{*}{0.001} \\
\hline & Minimal & $42.8 \%$ & $47.4 \%$ & $46.9 \%$ & \\
\hline
\end{tabular}


Table 1 The clinical characteristics of CHD children (including demographic characteristics, pre-operative color doppler echocardiography characteristics, the characteristic of surgery and cardiopulmonary bypass, and post-operative characteristics) (Continued)

\begin{tabular}{|c|c|c|c|c|c|}
\hline \multicolumn{2}{|l|}{ Parameter } & \multirow{2}{*}{$\begin{array}{l}\operatorname{LCOS}(n=864) \\
40.3 \%\end{array}$} & \multirow{2}{*}{$\begin{array}{l}\text { non-LCOS }(n=7796) \\
43.3 \%\end{array}$} & \multirow{2}{*}{$\begin{array}{l}\text { Total }(n=8660) \\
43 \%\end{array}$} & \multirow[t]{2}{*}{$P$ value } \\
\hline & Mild & & & & \\
\hline & Mild-to-moderate & $8.5 \%$ & $5.6 \%$ & $5.8 \%$ & \\
\hline & Moderate & $4.4 \%$ & $2.4 \%$ & $2.6 \%$ & \\
\hline & Moderate-to-severe & $2.0 \%$ & $0.4 \%$ & $0.6 \%$ & \\
\hline & Severe & $0.8 \%$ & $0.5 \%$ & $0.6 \%$ & \\
\hline \multirow[t]{3}{*}{ LVEF } & $<$ cut off value & $56.3 \%$ & $55.4 \%$ & $55.5 \%$ & 0.7 \\
\hline & Normal & $27.3 \%$ & $28.7 \%$ & $28.5 \%$ & \\
\hline & $>$ cut off value & $16.5 \%$ & $15.9 \%$ & $16 \%$ & \\
\hline \multirow[t]{3}{*}{ LVFS } & $<$ cut off value & $56.9 \%$ & $54.2 \%$ & $54.4 \%$ & 0.1 \\
\hline & Normal & $27.5 \%$ & $30.9 \%$ & $30.6 \%$ & \\
\hline & $>$ cut off value & $15.6 \%$ & $14.9 \%$ & $15.0 \%$ & \\
\hline \multirow[t]{2}{*}{ Mitral insufficiency } & NO & $92.0 \%$ & $93.8 \%$ & $93.6 \%$ & 0.044 \\
\hline & YES & $8.0 \%$ & $6.2 \%$ & $6.4 \%$ & \\
\hline \multirow[t]{2}{*}{ Mitral Stenosis } & NO & $99.0 \%$ & $99.4 \%$ & $99.4 \%$ & 0.1 \\
\hline & YES & $1.0 \%$ & $0.6 \%$ & $0.6 \%$ & \\
\hline \multicolumn{6}{|c|}{ The characteristic of surgery and cardiopulmonary bypass } \\
\hline \multirow[t]{2}{*}{ History of heart surgery } & NO & $84.6 \%$ & $92.3 \%$ & $91.5 \%$ & $<0.001$ \\
\hline & YES & $15.4 \%$ & $7.7 \%$ & $8.5 \%$ & \\
\hline \multirow[t]{6}{*}{ RACHS-1 risk grade } & 1 & $6.0 \%$ & $13.9 \%$ & $13.1 \%$ & $<0.001$ \\
\hline & 2 & $50.1 \%$ & $62.8 \%$ & $61.6 \%$ & \\
\hline & 3 & $32.4 \%$ & $20.9 \%$ & $22.1 \%$ & \\
\hline & 4 & $10.3 \%$ & $2.2 \%$ & $3.0 \%$ & \\
\hline & 5 & $1.2 \%$ & $0.1 \%$ & $0.2 \%$ & \\
\hline & 6 & $0 \%$ & $0.1 \%$ & $0.1 \%$ & \\
\hline CPB duration (min) & Median (IQR) & $87(62,119)$ & $51(38,72)$ & $53(39,78)$ & $<0.001$ \\
\hline Aortic clamping time (min) & Median (IQR) & $53(37,74)$ & $29(20,44)$ & $31(21,48)$ & $<0.001$ \\
\hline \multirow[t]{5}{*}{ Operation characteristics } & Selective extracorporeal surgery & $92.8 \%$ & $98.6 \%$ & $98 \%$ & $<0.001$ \\
\hline & Emergency non-extracorporeal surgery & $0.1 \%$ & $0.01 \%$ & $0.02 \%$ & \\
\hline & Emergency extracorporeal surgery & $7.1 \%$ & $1.2 \%$ & $1.8 \%$ & \\
\hline & Selective non-extracorporeal surgery & $0 \%$ & $0.1 \%$ & $0.1 \%$ & \\
\hline & Elective thoracotomy & $0 \%$ & $0.1 \%$ & $0.1 \%$ & \\
\hline \multirow[t]{4}{*}{ Circulating temperature } & Normal temperature & $21.8 \%$ & $53.1 \%$ & $49.9 \%$ & $<0.001$ \\
\hline & Mild hypothermia & $52.0 \%$ & $38.7 \%$ & $40.1 \%$ & \\
\hline & Deep hypothermia & $2.2 \%$ & $0.6 \%$ & $0.7 \%$ & \\
\hline & Middle and low temperature & $24.1 \%$ & $7.6 \%$ & $9.3 \%$ & \\
\hline \multirow[t]{6}{*}{ Circulation method } & Low flow cycle & $0.7 \%$ & $0.1 \%$ & $0.1 \%$ & $<0.001$ \\
\hline & Cerebral perfusion & $5.3 \%$ & $1.5 \%$ & $1.9 \%$ & \\
\hline & Parallel CPB & $3.0 \%$ & $3.4 \%$ & $3.3 \%$ & \\
\hline & Full flow & $89.9 \%$ & $94.6 \%$ & $94.2 \%$ & \\
\hline & Circulatory arrest & $0.9 \%$ & $0.4 \%$ & $0.4 \%$ & \\
\hline & Cerebrovascular perfusion & $0.1 \%$ & $0.1 \%$ & $0.1 \%$ & \\
\hline Difficult to wean from CPB & NO & $99.7 \%$ & $99.9 \%$ & $99.9 \%$ & 0.1 \\
\hline
\end{tabular}


Table 1 The clinical characteristics of CHD children (including demographic characteristics, pre-operative color doppler echocardiography characteristics, the characteristic of surgery and cardiopulmonary bypass, and post-operative characteristics) (Continued)

\begin{tabular}{|c|c|c|c|c|c|}
\hline Parameter & & $\operatorname{LCOS}(n=864)$ & non-LCOS $(n=7796)$ & Total $(n=8660)$ & $P$ value \\
\hline & YES & $0.4 \%$ & $0.1 \%$ & $0.1 \%$ & \\
\hline \multirow[t]{2}{*}{ Myocardial preservation } & HTK & $31.6 \%$ & $8.9 \%$ & $11.2 \%$ & $<0.001$ \\
\hline & Cold blood cardioplegia (4:1) & $68.4 \%$ & $91.1 \%$ & $88.8 \%$ & \\
\hline \multicolumn{6}{|l|}{ Post-operative characteristic } \\
\hline \multirow[t]{2}{*}{ Residual shunt } & NO & $98.4 \%$ & $99.6 \%$ & $99.5 \%$ & $<0.001$ \\
\hline & YES & $1.62 \%)$ & $0.4 \%$ & $0.5 \%$ & \\
\hline \multirow[t]{2}{*}{ LVOTO } & NO & $98.3 \%$ & $99.5 \%$ & $99.4 \%$ & $<0.001$ \\
\hline & YES & $1.7 \%$ & $0.5 \%$ & $0.6 \%$ & \\
\hline \multirow[t]{2}{*}{ RVOTO } & NO & $98.4 \%$ & $99.7 \%$ & $99.6 \%$ & $<0.001$ \\
\hline & YES & $1.6 \%$ & $0.3 \%$ & $0.4 \%$ & \\
\hline \multirow[t]{5}{*}{ Postoperative rhythm of the heart } & $\| I^{\circ} A V B$ & $1.2 \%$ & $0.1 \%$ & $0.2 \%$ & $<0.001$ \\
\hline & $\|{ }^{\circ} A \vee B$ & $0.6 \%$ & $0.2 \%$ & $0.2 \%$ & \\
\hline & Atrioventricular dissociation & $0.2 \%$ & $0.1 \%$ & $0.1 \%$ & \\
\hline & Supraventricular tachycardia & $0.2 \%$ & $0.1 \%$ & $0.1 \%$ & \\
\hline & Sinus rhythm & $97.8 \%$ & $99.5 \%$ & $99.3 \%$ & \\
\hline
\end{tabular}

Abbreviation: CHD Congenital Heart Disease, LCOS Low Cardiac Output Syndrome, BMI Body Mass Index, Al Aortic Insufficiency, MR Mitral Regurgitation, PI Pulmonary Insufficiency, TR Tricuspid Regurgitation, LVEF Left Ventricular Ejection Fraction, LVFS left ventricular fraction shortening, CPB Cardiopulmonary Bypass, LVOTO Left Ventricular Outflow Tract Obstruction, RVOTO Right Ventricular Outflow Tract Obstruction, HTK histidine-tryptophan-ketoglutarate, AVB atrioventricular block

LCOS occurred in 32 of 76 (42\%) neonates (median age, 7 days) after cardiac surgery [17]. This difference was attributed to the probable difference in age. Neonatal myocardium is physiologically immature [18-21] and every procedure affects the incidence of LCOS differently [22]. The age range of children included in this study was larger (1-6457 days), and we included different types of cardiac surgery. This could be the main reason why the LCOS incidence was higher than that in adults and lower than that in newborns.

We found that age, aortic shunt (left-to-right and bidirectional), atrial shunt, ventricular level shunt (left-toright and bi-directional), circulation temperature, and myocardial preservation using HTK were independent predictors of LCOS.

The risk for LCOS was higher for neonates given that their myocardial development is not complete. The effect of age on the risk of LCOS in adults has been confirmed earlier. In a prospective study, multivariate analysis showed that age was an independent predictor of LCOS [9] and a retrospective study ascertained that increasing age is an independent predictor of LCOS [2].

By preoperative Doppler echocardiography characteristics of CHD children, aortic shunt (left-to-right and bi-directional), atrial shunt, ventricular level shunt (left-to-right and bi-directional) were deduced as significant risk factors for LCOS. Left-to-right shunt before surgery may result in right heart failure, leading to low cardiac output. Xiong et al. found that preoperative right-left shunt ventricular septal defect was a risk factor of LCOS [23]. Ischemic mitral valve pathology was found as an independent predictor for LCOS after isolated mitral valve surgery [3].

We also found that the circulation temperature (mild hypothermia, middle and low temperature) and the proportion of myocardium preserved using HTK were independent predictors of LCOS. During routine hypothermic $\mathrm{CPB}$, the heart is damaged by hypothermia, ischemiareperfusion, or hyperkalemia, which is associated with an excess risk of postoperative LCOS and severe arrhythmia [24]. Yau et al. found that during hypothermic CPB, the myocardium acquires energy through anaerobic metabolism, leading to the accumulation of lactic acid. After reperfusion, aerobic metabolism cannot be resumed immediately in cardiomyocytes owing to low temperature, but still anaerobic metabolism can occur for a short period. Whereas, patients with mild hypothermia CPB are less affected [25]. The study found that the inflammatory response mediated by CPB caused pulmonary vascular endothelial damage, which changed the pulmonary vascular reactivity. These cause excessive thromboxane production and reduced endogenous nitric oxide production, which can lead to pulmonary vasoconstriction and formation of pulmonary microthrombus. Further, these changes could induce pulmonary vascular resistance, which 
Table 2 Univariate/multivariate logistic regression analysis for the association of pre-/post-operative characteristics with LCOS

\begin{tabular}{|c|c|c|c|c|c|}
\hline \multirow[b]{2}{*}{ Parameter } & & \multicolumn{2}{|l|}{ Univariate analysis } & \multicolumn{2}{|l|}{ Multivariate analysis } \\
\hline & & OR $(95 \% \mathrm{Cl})$ & $P$-value & OR $(95 \% \mathrm{Cl})$ & Pvalue \\
\hline Age (days) & & $0.992(0.989,0.995)$ & $<0.001$ & $0.992(0.988,0.997)$ & 0.001 \\
\hline Gender & Male vs Female & $1.06(0.92,1.22)$ & 0.4 & & \\
\hline $\mathrm{Al}$ & & $1.118(1.008,1.239)$ & 0.034 & & \\
\hline $\mathrm{MR}$ & & $1.06(0.99,1.14)$ & 0.1 & & \\
\hline PI & & $0.845(0.751,0.951)$ & 0.005 & & \\
\hline $\mathrm{TR}$ & & $1.226(1.13,1.33)$ & $<0.001$ & $1.192(1.072,1.326)$ & 0.001 \\
\hline RACHS-1 risk grade & & $2.094(1.903,2.304)$ & $<0.001$ & $1.166(1.011,1.345)$ & 0.035 \\
\hline \multirow[t]{3}{*}{ Aortic shunt } & Left-to-right vs None & $2.363(1.904,2.932)$ & $<0.001$ & $1.37(1.005,1.867)$ & 0.046 \\
\hline & Right-to-left vs None & $27.469(5.318,141.869)$ & $<0.001$ & & \\
\hline & Bi-directional vs None & $5.087(3.721,6.953)$ & $<0.001$ & $1.716(1.138,2.587)$ & 0.01 \\
\hline \multirow[t]{3}{*}{ Atrial shunt } & Left-to-right vs None & $1.396(1.151,1.693)$ & 0.001 & $1.407(1.097,1.805)$ & 0.007 \\
\hline & Right-to-left vs None & $4.412(3.149,6.18)$ & $<0.001$ & $3.168(1.944,5.163)$ & $<0.001$ \\
\hline & Bi-directional vs None & $4.111(3.34,5.059)$ & $<0.001$ & $1.87(1.389,2.519)$ & $<0.001$ \\
\hline \multirow[t]{3}{*}{ Ventricular level shunt } & Left-to-right vs None & $0.37(0.288,0.476)$ & $<0.001$ & $0.676(0.486,0.94)$ & 0.02 \\
\hline & Right-to-left vs None & $3.16(0.65,15.32)$ & 0.2 & $3.458(0.452,26.448)$ & 0.2 \\
\hline & Bi-directional vs None & $2.452(2.052,2.93)$ & $<0.001$ & $2.09(1.611,2.712)$ & $<0.001$ \\
\hline \multirow[t]{4}{*}{ Operation characteristics } & $\begin{array}{l}\text { Emergency non-extracorporeal surgery } \\
\text { vs Selective extracorporeal surgery }\end{array}$ & $9.58(0.6153 .34)$ & 0.1 & & \\
\hline & $\begin{array}{l}\text { Emergency extracorporeal surgery vs } \\
\text { Selective extracorporeal surgery }\end{array}$ & $6.153(4.425,8.556)$ & $<0.001$ & & \\
\hline & $\begin{array}{l}\text { Selective non-extracorporeal surgery } \\
\text { vs Selective extracorporeal surgery }\end{array}$ & $0(0,1.053157 \mathrm{E} 255)$ & 0.97 & & \\
\hline & $\begin{array}{l}\text { Elective thoracotomy vs Selective } \\
\text { extracorporeal surgery }\end{array}$ & $0(0)$ & 0.98 & & \\
\hline History of heart surgery & YES vS NO & $2.17(1.772,2.658)$ & $<0.001$ & & \\
\hline Residual shunt & YES vs NO & $4.264(2.252,8.073)$ & $<0.001$ & $3.489(1.502,8.105)$ & 0.004 \\
\hline LVOTO & YES vS NO & $3.81(2.078,6.988)$ & $<0.001$ & $3.934(1.673,9.254)$ & 0.002 \\
\hline RVOTO & YES vs NO & $5.82(2.967,11.418)$ & $<0.001$ & $3.638(1.225,10.798)$ & 0.02 \\
\hline \multirow[t]{3}{*}{ Circulating temperature } & Mild hypothermia vs Normal temperature & $3.271(2.741,3.903)$ & $<0.001$ & $1.526(1.205,1.934)$ & $<0.001$ \\
\hline & Deep hypothermia vs Normal temperature & $9.289(5.328,16.194)$ & $<0.001$ & $1.369(0.587,3.194)$ & 0.467 \\
\hline & $\begin{array}{l}\text { Middle and low temperature vs Normal } \\
\text { temperature }\end{array}$ & $7.691(6.202,9.537)$ & $<0.001$ & $1.738(1.236,2.443)$ & 0.001 \\
\hline \multirow[t]{5}{*}{ Circulation method } & Low flow vs Full flow & $11.395(3.47,37.422)$ & $<0.001$ & & \\
\hline & Cerebral perfusion vs Full flow & $3.671(2.591,5.199)$ & $<0.001$ & & \\
\hline & Parallel CPB vs Full flow & $0.94(0.63,1.42)$ & 0.8 & & \\
\hline & Circulatory arrest vs Full flow & $2.813(1.274,6.214)$ & 0.011 & & \\
\hline & Cardio-cerebral Perfusion vs Full flow & $1.90(0.22,16.28)$ & 0.6 & & \\
\hline CPB duration (min) & & $1.016(1.014,1.017)$ & $<0.001$ & $1.009(1.006,1.012)$ & $<0.001$ \\
\hline Aortic clamping time (min) & & $1.026(1.023,1.028)$ & $<0.001$ & & \\
\hline \multirow[t]{4}{*}{ Postoperative rhythm of the heart } & \|\|$^{\circ} A V B$ vs Sinus rhythm & $9.181(3.81,22.121)$ & $<0.001$ & & \\
\hline & $\|^{\circ} A V B$ vs Sinus rhythm & $2.869(1.048,7.851)$ & 0.04 & & \\
\hline & Atrioventricular dissociation vs Sinus rhythm & $2.30(0.49,10.83)$ & 0.3 & & \\
\hline & Supraventricular tachycardia vs Sinus rhythm & $4.59(0.84,25.1)$ & 0.1 & & \\
\hline Difficult to wean from CPB & YES vs NO & $4.524(1.129,18.121)$ & 0.033 & & \\
\hline Myocardial preservation & HTK vs Cold blood cardioplegia (4:1) & $4.744(4.014,5.607)$ & $<0.001$ & $1.677(1.298,2.167)$ & $<0.001$ \\
\hline
\end{tabular}


Table 2 Univariate/multivariate logistic regression analysis for the association of pre-/post-operative characteristics with LCOS (Continued)

\begin{tabular}{|c|c|c|c|c|c|}
\hline & & Univariate analysis & & Multivariate analysis & \\
\hline \multirow[t]{2}{*}{$\overline{\mathrm{BMI}}$} & $<5$ th percentile vs 5 th $\sim 5$ th percentile & $1.305(1.086,1.569)$ & 0.004 & & \\
\hline & $>95$ th percentile vs 5 th 5 th percentile & $1.03(0.82,1.28)$ & 0.8 & & \\
\hline \multirow[t]{2}{*}{ LVEF } & $<$ cut off value vs Normal & $1.07(0.90,1.27)$ & 0.4 & & \\
\hline & $>$ cut off value vs Normal & $1.09(0.87,1.37)$ & 0.5 & & \\
\hline \multirow[t]{2}{*}{ LVFS } & $<$ cut off value vs Normal & $1.18(1.00,1.4)$ & 0.1 & & \\
\hline & $>$ cut off value vs Normal & $1.18(0.93,1.48)$ & 0.2 & & \\
\hline Mitral insufficiency & YES VS NO & $1.309(1.006,1.702)$ & 0.045 & $1.714(1.239,2.37)$ & 0.001 \\
\hline Mitral stenosis & YES VS NO & $1.77(0.87,3.64)$ & 0.1 & & \\
\hline
\end{tabular}

Abbreviation: OR Odds Ratio, Cl Confidence Interval, LCOS Low Cardiac Output Syndrome, BMI Body Mass Index, Al Aortic Insufficiency, MR Mitral Regurgitation, PI Pulmonary Insufficiency, TR Tricuspid Regurgitation, LVEF Left Ventricular Ejection Fraction, LVFS left ventricular fraction shortening, CPB Cardiopulmonary Bypass, LVOTO Left Ventricular Outflow Tract Obstruction, RVOTO Right Ventricular Outflow Tract Obstruction, HTK histidine-tryptophan-ketoglutarate, AVB atrioventricular block

increases after $\mathrm{CPB}$ [26]. Then, the right ventricular afterload increases, which is significantly associated with right ventricular dysfunction and LCOS.

Obesity or high BMI is a common factor associated with poor prognosis. A propensity score-matched analysis found that obese (BMI, $\geq 30 \mathrm{~kg} / \mathrm{m}^{2}$ ) patients who underwent surgery for type A acute aortic dissection had higher postoperative mortality rates. Moreover, a previous report stated that obesity was significantly associated with increased risk of LCOS and other postoperative morbidities [27]. However, in our study, there was no significant correlation between BMI and LCOS risk after cardiac surgery in CHD children. This correlation is different from the results of the abovementioned study on adults and we ascertain that the 'obesity paradox' may explain this inconsistency. The BMI of the CHD children in our study may be lower than that of the general population, and the patients with $\mathrm{BMI}>\mathrm{P} 95$ percentile did not have such high obesity levels.

LCOS is associated with significantly high morbidity and mortality. In our study, the mortality rate $(7.18 \%$ vs. $1.08 \%, p<0.001)$, ICU stay $>3$ days $(89.24 \%$ vs.

Table 3 The post-operative mortality, length of ICU $>3 d$, duration of Medical ventilator $>48 \mathrm{~h}$ in LCOS and non-LCOS CHD children

\begin{tabular}{llllll}
\hline Parameter & & $\begin{array}{l}\text { LCOS } \\
(n=864)\end{array}$ & $\begin{array}{l}\text { Non- LCOS } \\
(n=7796)\end{array}$ & $\begin{array}{l}\text { Total } \\
(n=8660)\end{array}$ & $P$ value \\
\hline Mortality (\%) & NO & $92.8 \%$ & $98.9 \%$ & $98.3 \%$ & $<0.001$ \\
& YES & $7.2 \%$ & $1.1 \%$ & $1.7 \%$ & \\
Length of & $<=3 \mathrm{~d}$ & $10.8 \%$ & $66.7 \%$ & $61.1 \%$ & $<0.001$ \\
ICU stay & $>3 \mathrm{~d}$ & $89.2 \%$ & $33.4 \%$ & $38.9 \%$ & \\
$\begin{array}{l}\text { Duration of } \\
\text { Medical ventilator }\end{array}$ & $<=48 \mathrm{~h}$ & $6.8 \%$ & $55.5 \%$ & $50.7 \%$ & $<0.001$ \\
& $>48 \mathrm{~h}$ & $93.2 \%$ & $44.5 \%$ & $49.3 \%$ & \\
\hline
\end{tabular}

Abbreviation: ICU Intensive Care Unit, LCOS Low Cardiac Output Syndrome, CHD Congenital Heart Disease
$33.35 \%, p<0.001)$ and the duration of mechanical ventilator support $>48 \mathrm{~h}(93.21 \%$ vs. $44.5 \%, p<0.001)$ were significantly higher in LCOS children compared to those without LCOS. A previous study showed that LCOS was associated with significantly high morbidity and mortality in adults [3].

Therefore, cardiac surgery can cause LCOS in children with CHD and result in poorer postsurgical outcomes. In the current study, we confirmed the impact of LCOS on postoperative clinical outcomes in CHD children.

\section{Limitations}

This study had certain limitations. First, this is a retrospective study; the correlations may not confirm a causal relationship between the risk factors and LCOS. Second, we included patients from a single center only; hence, the application of these findings on other populations and institutions need to be reproduced in further large-scale multi-center studies. Since this is a single-center study, the impact of surgery outcomes and hospitalizations from other centers was not considered.

Table 4 Incidence of postoperative LCOS in children with CHD of different nutritional status

\begin{tabular}{llll}
\hline Age & $\begin{array}{l}\text { BMI }<5 \text { th } \\
\text { percentile }\end{array}$ & $\begin{array}{l}\text { BMI 5th 95th } \\
\text { percentile }\end{array}$ & $\begin{array}{l}\text { BMI > 95th } \\
\text { percentile }\end{array}$ \\
\hline$<1$ month & $41.2 \%$ & $39.4 \%$ & $35.7 \%$ \\
1 month 3 month & $17.9 \%$ & $26.1 \%$ & $17.9 \%$ \\
3 month 6 month & $21.4 \%$ & $12.5 \%$ & $9.1 \%$ \\
6 month 1 year & $11.5 \%$ & $9.3 \%$ & $5.1 \%$ \\
1 year 3 year & $10.2 \%$ & $4.4 \%$ & $4.2 \%$ \\
$>$ 3 year & $12.6 \%$ & $7.8 \%$ & $3.1 \%$ \\
\hline
\end{tabular}

Abbreviation: 3.LCOS Low Cardiac Output Syndrome, CHD Congenital Heart Disease 


\section{Conclusion}

In conclusion, our study provides clinically significant evidence to indicate a significant association of LCOS with postoperative clinical outcomes (including mortality, prolonged ICU stay, and extended mechanical ventilator support) in children with CHD. Circulation temperature, myocardial preservation using HTK, and usage of residual shunt after surgery were independent risk predictors for LCOS. Further multi-center studies toned to be conducted with a larger sample size to confirm our study results.

\section{Supplementary information}

Supplementary information accompanies this paper at https://doi.org/10. 1186/s12887-020-1972-y.

Additional file 1: Table S1. The Variance Inflation Factor of selected variables.

\section{Abbreviations \\ BMI: Body mass index; CHD: Congenital heart disease; CPB: Cardiopulmonary bypass; HTK: Histidine-tryptophan-ketoglutarate; ICU: Intensive care unit; LCOS: Low cardiac output syndrome; LVEF: Left ventricular ejection fraction; LVFS: Left ventricular fractional shortening; LVOTO: Left ventricular outflow tract obstruction; MR: Mitral regurgitation; RACHS-1: Risk Adjustment in Congenital Heart Surgery-1; RVOTO: Right ventricular outflow tract obstruction; TR: Tricuspid regurgitation}

\section{Acknowledgments}

Extremely grateful to all the staff who contributed to the study. Thankful to Shanghai Synyi Medical Technology Co., Ltd. for providing the data analysis and statistical platform.

\section{Authors' contributions}

XD acquired the data, implemented the research, revised the article, and approved the final version of the manuscript for publication. HC was responsible for data acquisition, data cleaning, analysis and interpretation of data, and finalizing the manuscript for publication. XS was responsible for data review, data analysis, manuscript revision, and finalizing the manuscript for publication. SW was responsible for supervision of the project execution (ensuring the accuracy or integrity of any part of the work), acquisition of data, data analysis and interpretation, approving the final version of the manuscript to be published, and agreed to be accountable for all aspects of the work in ensuring that questions related to the accuracy or integrity of any part of the work are appropriately investigated and resolved. ZH was responsible for analysis and interpretation of data, drafting the article, and approving the final manuscript to be published. LY was responsible for the statistical analysis of the research data, revising the article, and approving the final manuscript to be published. ZL was responsible for the determination of the research direction, the design of the research program, summarizing the research questions, analysis, data interpretation, final approval of the version to be published, and agreed to be accountable for all aspects of the work in ensuring that questions related to the accuracy or integrity of any part of the work are appropriately investigated and resolved. The author(s) read and approved the final manuscript

\section{Funding}

The research was funded by the Fund of Shanghai Jiao Tong University (No: YG2015QN23).

\section{Availability of data and materials}

The datasets generated during and/or analyzed during the current study are not publicly available due to the hospital regulation.

\section{Ethics approval and consent to participate}

Our retrospective study is in accordance with the ethical principles of the "Declaration of Helsinki" and the "International Ethical Guidelines for Biomedical Research Involving Human Subjects" enforced by the Council for International Organizations of Medical Science. Therefore, the ethical committee of the hospital decided to exempt the study from informed consent.

\section{Consent for publication}

Not applicable.

\section{Competing interests}

The authors declare that they have no competing interests.

\section{Author details}

'Department of Cardiothoracic Surgery, Shanghai Children's Medical Center, School of Medicine, Shanghai Jiao Tong University, 1678 Dongfang Road, Pudong district, Shanghai, China. ${ }^{2}$ Synyi Medical Technology, Shanghai, China.

Received: 11 June 2019 Accepted: 11 February 2020

Published online: 24 February 2020

\section{References}

1. Yuerek M, Rossano JW, Mascio CE, Shaddy RE. Postoperative management of heart failure in pediatric patients. Expert Rev Cardiovasc Ther. 2016;14(2): 201-15.

2. Maganti MD, Rao V, Borger MA, Ivanov J, David TE. Predictors of low cardiac output syndrome after isolated aortic valve surgery. Circulation. 2005;112(9 Suppl):1448-52.

3. Maganti M, Badiwala M, Sheikh A, Scully H, Feindel C, David TE, Rao V. Predictors of low cardiac output syndrome after isolated mitral valve surgery. J Thorac Cardiovasc Surg. 2010;140(4):790-6.

4. Masse L, Antonacci M. Low cardiac output syndrome: identification and management. Crit Care Nurs Clin North Am. 2005;17(4):375-83 x.

5. Hoffman TM, Wernovsky G, Atz AM, Bailey JM, Akbary A, Kocsis JF, Nelson DP, Chang AC, Kulik TJ, Spray TL, et al. Prophylactic intravenous use of milrinone after cardiac operation in pediatrics (PRIMACORP) study. Prophylactic intravenous use of Milrinone after cardiac operation in pediatrics. Am Heart J. 2002;143(1):15-21.

6. Rao V, Ivanov J, Weisel RD, Ikonomidis JS, Christakis GT, David TE. Predictors of low cardiac output syndrome after coronary artery bypass. J Thorac Cardiovasc Surg. 1996;112(1):38-51.

7. Algarni KD, Maganti M, Yau TM. Predictors of low cardiac output syndrome after isolated coronary artery bypass surgery: trends over 20 years. Ann Thorac Surg. 2011;92(5):1678-84.

8. Nashef SA, Roques F, Sharples LD, Nilsson J, Smith C, Goldstone AR, Lockowandt U. EuroSCORE II. Eur J Cardio-Thorac Surg. 2012;41(4):734-44 discussion 744-735.

9. Balderas-Munoz K, Rodriguez-Zanella H, Fritche-Salazar JF, Avila-Vanzzini N, Juarez Orozco LE, Arias-Godinez JA, Calvillo-Arguelles O, Rivera-Peralta S, Sauza-Sosa JC, Ruiz-Esparza ME, et al. Improving risk assessment for postsurgical low cardiac output syndrome in patients without severely reduced ejection fraction undergoing open aortic valve replacement. The role of global longitudinal strain and right ventricular free wall strain. Int J Cardiovascular Imaging. 2017;33(10):1483-9.

10. Ding W, Ji Q, Shi Y, Ma R. Predictors of low cardiac output syndrome after isolated coronary artery bypass grafting. Int Heart J. 2015;56(2):144-9.

11. Sa MP, Nogueira JR, Ferraz PE, Figueiredo OJ, Cavalcante WC, Cavalcante TC, Silva HT, Santos CA, Lima RO, Vasconcelos FP, et al. Risk factors for low cardiac output syndrome after coronary artery bypass grafting surgery. Revista brasileira de cirurgia cardiovascular : orgao oficial da Sociedade Brasileira de Cirurgia Cardiovascular. 2012;27(2):217-23.

12. Burkhardt BE, Rucker G, Stiller B. Prophylactic milrinone for the prevention of low cardiac output syndrome and mortality in children undergoing surgery for congenital heart disease. Cochrane Database Syst Rev. 2015;3:Cd009515.

13. Qu J, Liang H, Zhou N, Li L, Wang Y, Li J, Cui Y. Perioperative NT-proBNP level: potential prognostic markers in children undergoing congenital heart disease surgery. J Thorac Cardiovasc Surg. 2017;154(2):631-40.

14. Li H, Ji C, Zong X, Zhang Y. Body mass index growth curves for Chinese children and adolescents aged 0 to 18 years. Zhonghua Er Ke Za Zhi. 2009; 47(7):493-8 
15. Cavalcante $C T$, de NMG S, Júnior PVC, Branco KM, Pompeu RG, Teles AC, Cavalcante RC, de Andrade GV. Analysis of Surgical Mortality for Congenital Heart Defects Using RACHS-1Risk Score in a Brazilian Single Center. Brazilian J Cardiovascular Surg. 2016;30(3):1 219-225.

16. Hoffman TM, Wernovsky G, Atz AM, Kulik TJ, Nelson DP, Chang AC, Bailey JM, Akbary A, Kocsis JF, Kaczmarek R, et al. Efficacy and safety of milrinone in preventing low cardiac output syndrome in infants and children after corrective surgery for congenital heart disease. Circulation. 2003;107(7):996-1002.

17. Butts RJ, Scheurer MA, Atz AM, Zyblewski SC, Hulsey TC, Bradley SM, Graham EM. Comparison of maximum vasoactive inotropic score and low cardiac output syndrome as markers of early postoperative outcomes after neonatal cardiac surgery. Pediatr Cardiol. 2012;33(4):633-8.

18. Balaguru D, Haddock PS, Puglisi JL, Bers DM, Coetzee WA, Artman M. Role of the sarcoplasmic reticulum in contraction and relaxation of immature rabbit ventricular myocytes. J Mol Cell Cardiol. 1997;29(10):2747-57.

19. Hatem SN, Sweeten T, Vetter V, Morad M. Evidence for presence of Ca2+ channel-gated $\mathrm{Ca} 2+$ stores in neonatal human atrial myocytes. Am J Phys. 1995;268(3 Pt 2):H1195-201.

20. Maylie JG. Excitation-contraction coupling in neonatal and adult myocardium of cat. Am J Phys. 1982;242(5):H834-43.

21. Nassar R, Reedy MC, Anderson PA. Developmental changes in the ultrastructure and sarcomere shortening of the isolated rabbit ventricular myocyte. Circ Res. 1987;61 (3):465-83.

22. Lomivorotov W, Efremov SM, Kirov MY, Fominskiy EV, Karaskov AM. Lowcardiac-output syndrome after cardiac surgery. J Cardiothorac Vasc Anesth. 2017;31(1):291-308.

23. LQ-s XIONG H-h, Xiao-hua XU, et al. Studying the velocities and timings across the Large VSD to predict the surgery effect of tetralogy of fallot by color M-mode and color flow Imaging. cta Academiae Medicinae Jiangxi. 2008;48(4):65-70

24. Warner KG, Khuri SF, Kloner RA, Josa M, Dalecki-Chipperfield KM, Butler MD, Assousa SN, Lee SS, Barsamian EM, Seiler M. Structural and metabolic correlates of cell injury in the hypertrophied myocardium during valve replacement. J Thorac Cardiovasc Surg. 1987;93(5):741-54.

25. Yau TM, Ikonomidis JS, Weisel RD, Mickle DA, Ivanov J, Mohabeer MK, Tumiati L, Carson S, Liu P. Ventricular function after normothermic versus hypothermic cardioplegia. J Thorac Cardiovasc Surg. 1993;105(5):833-43 discussion 843-834.

26. Lam CF, Peterson TE, Croatt AJ, Nath KA, Katusic ZS. Functional adaptation and remodeling of pulmonary artery in flow-induced pulmonary hypertension. Am J Phys Heart Circ Phys. 2005;289(6):H2334-41.

27. Lio A, Bovio E, Nicolò F, Saitto G, Scafuri A, Bassano C, Chiariello L, Ruvolo G: Influence of Body Mass Index on Outcomes of Patients Undergoing Surgery for Acute Aortic Dissection: A Propensity-Matched Analysis. Tex Heart Inst J 2019;46(1): $1-13$.

\section{Publisher's Note}

Springer Nature remains neutral with regard to jurisdictional claims in published maps and institutional affiliations.

Ready to submit your research? Choose BMC and benefit from:

- fast, convenient online submission

- thorough peer review by experienced researchers in your field

- rapid publication on acceptance

- support for research data, including large and complex data types

- gold Open Access which fosters wider collaboration and increased citations

- maximum visibility for your research: over $100 \mathrm{M}$ website views per year

At BMC, research is always in progress.

Learn more biomedcentral.com/submissions 